\title{
Impacto de la resignificación de la práctica pedagógica investigativa y del currículo de graduados de pedagogía de instituciones de educación superior en Barranquilla-Colombia
}

\author{
Hilda R. Guerrero-Cuentas ${ }^{(1)^{\star}}$, Yuneidis Morales-Ortega ${ }^{(2)}$; Greys P. Nuñez-Rios ${ }^{(1)}$ y Elias D. Medina-Fonseca ${ }^{(1)}$ \\ (1) Facultad de Ciencias Humanas y Sociales, Departamento de Humanidades, Universidad de la Costa, Calle 58 \# 55 - \\ 66. Barranquilla, Colombia (correo-e: hguerrero@cuc.edu.co; gnunez4@cuc.edu.co; emedina14@cuc.edu.co) \\ (2) Facultad de Ciencias Económicas, Departamento de Ciencias Empresariales, Universidad de la Costa, Calle 58 \# 55 \\ - 66. Barranquilla, Colombia (correo-e: ymorales4@cuc.edu.co)
}

* Autor a quien debe ser dirigida la correspondencia

Recibido Jun. 21, 2019; Aceptado Ago. 20, 2019; Versión final Oct. 8, 2019, Publicado Abr. 2020

\section{Resumen}

Este trabajo tiene como objetivo indagar sobre cómo el graduado universitario de pedagogía asume desde el ejercicio de su rol profesional la resignificación de la práctica pedagógica investigativa. Se evalúa el impacto de los graduados del programa de Especialización en Estudios Pedagógicos de la Universidad de la Costa (Colombia) durante el periodo 2014-2017, frente a la resignificación de la práctica pedagógica investigativa. El estudio se fundamentó los preceptos y lineamentos planteados por el Consejo Nacional de Acreditación y el proyecto Tuning. Se trabajó bajo un enfoque cuantitativo con una población de 247 egresados, utilizando la técnica de la observación y el cuestionario de auto reporte como instrumento para la recolección de información. El estudio muestra que el trabajo curricular desarrollado en el programa impacta positivamente el desempeño de los egresados en el mercado laboral. Se evidencia así la pertinencia del programa en su formación profesional y desempeño laboral al ajustarse los planes curriculares acorde a las necesidades nacionales e internacionales.

\section{Impact of the resignification of the research pedagogical practice and the curriculum of pedagogy degree of higher education institutions in Barranquilla-Colombia}

\begin{abstract}
This work has the objective of investigating how the pedagogy university graduate assume, from the exercise of his professional role, the resignification of the pedagogical research practice. The impact of graduates of the Specialization Program in Pedagogical Studies of the University of the Coast during the 2014-2017 period, on the resignification of the research pedagogical practice is evaluated. The study was based on the precepts and guidelines proposed by the National Accreditation Council and the Tuning project. A quantitative approach with a population of 247 graduates was considered, using the observation technique and the self-report questionnaire as the instrument for gathering information. The study shows that the curricular work developed in the program positively impacts the performance of graduates in the labor market. This demonstrates the relevance of the program in their professional training and work performance by adjusting the curricular plans according to national and international needs.
\end{abstract}

Keywords: graduates; impact; pedagogical practice; curriculum; job performance 


\section{INTRODUCCIÓN}

El tema de los graduados, se ha convertido en los últimos años en una variable de relevada importancia para las Instituciones de Educación Superior - IES, tanto a nivel nacional como internacional, estas Instituciones por su parte, intentan desarrollar competencias laborales desde la mejora continua y la calidad de la educación, para que los graduados alcancen altos niveles de competencias laborales. Es así como el Proyecto Tuning para América Latina 2004-2007, es considerado por (Bravo, 2007) como una metodología implementada para la comprensión del currículo de una manera más comparable, donde el contenido de los estudios y su estructura se convierta en su principal exponente. Como parte de su metodología se introdujo el concepto de resultados del aprendizaje y competencias que involucran todos aquellos conocimientos y habilidades que se considera que los estudiantes comprendan, demuestren y dominen, una vez culminen su proceso de aprendizaje.

En la búsqueda de contribuir con el desarrollo de títulos comprensibles y comparables se implementó el Proyecto Tuning en 19 países (Argentina, Bolivia, Brasil, Chile, Colombia, Costa Rica, Cuba, Ecuador, El Salvador, Guatemala, Honduras, México, Nicaragua, Panamá, Paraguay, Perú, República Dominicana, Uruguay y Venezuela), en los 12 campos del saber tales como: Administración de Empresas, Arquitectura, Derecho, Educación, Historia, Geología, Enfermería, Física, Ingeniería Civil, Química, Matemáticas y Medicina, como búsqueda de facilitar la movilidad de profesiones con una alta capacidad de desempeño centrados en las competencias y desempeño basados en el conocimiento. Este estudio permitió hacer seguimiento al proceso hasta llegar al consenso de estudio de las 27 competencias genéricas, siendo éstas incorporadas a cualquier profesión y un acumulado de competencias específicas propias por cada 12 áreas estudiadas en el proyecto, quedando en discusión por parte de los gobiernos hacer control del tema, estudiar los perfiles profesionales, competencias genéricas y específicas, para las áreas de estudio (Beneitone et al., 2007, p. 34).

Por tanto, se observa la preocupación del Proyecto Tuning al plantear con países amigos proyectos que favorezcan a los graduados, aunando esfuerzos para fortalecer las competencias específicas y genéricas, que determinen la calidad del ser humano que se profesionaliza, para prestar sus servicios académicos, desarrollando de la mejor manera su saber específico. En este particular en Colombia, a través del Consejo Nacional de Acreditación (CNA, 2014) específicamente considerando sus lineamientos orientados a las Instituciones de Educación Superior, se pretende el mejoramiento de la calidad y el reconocimiento mediante estándares internacionales. De allí, la preocupación de la educación superior para formar profesionales en todas las áreas y campos de acción, capaces de enfrentar nuevos retos derivados a los avances científicostecnológicos y poseyendo un alto nivel de competencias que permitan reconocer y comprender las necesidades sociales y económicas que impacten directamente al país. En tal sentido, esto ha constituido un referente importante en el estudio, debido a que es el punto de partida para la comprensión de la ruta formativa y su pertinencia con el contexto.

Por su parte (Rojo y Navarro, 2016) plantean cómo las universidades al ser Instituciones de Educación Superior deben asegurar la calidad en la formación de sus profesionales, responsabilizándose en priorizar competencias específicas y humanas que aporten a la satisfacción de las necesidades de la población, contribuyendo al desarrollo social, económico y cultural de la sociedad. Es así como (Orozco et al., 2009) consideran que la calidad de la educación convoca a propósitos claros relacionados con la interpretación conceptual de los escenarios Institucionales, en la cual emergen las preocupaciones y compromisos sociales de la educación, mediado por el discurso político y pedagógico en la que conciben las instituciones educativas como contextos curriculares, para hacerle frente a las competencias laborales exigidas por un mercado ampliamente globalizado. En este sentido, las concepciones de competencias donde se compromete la vida laboral del graduado deja claro las responsabilidades con las cuales se va a encontrar en la organización y cómo este debe responder ante ellas, priorizando sus habilidades y destrezas profesionales que le permitan respaldar la imagen de él como trabajador, cumpliendo con las tareas laborales, que le fueron asignadas y responsabilizándose de ellas de la mejor manera, pero sobre todo respondiendo a las competencias laborales, por las cuales fue contratado en la organización.

En este orden de ideas (Abadía et al., 2015) explican que las competencias desarrolladas por los docentes están articuladas por el ejercicio de la práctica con sus estudiantes y éstas se ven reflejadas cuando los educadores aplican elementos evaluativos que impactan en sus aptitudes, habilidades, capacidades, destrezas y conocimientos; siendo una misión de cada docente conducir por el crecimiento tanto personal como profesional del estudiante, permitiendo distinguir su competitividad en el entorno empresarial y social.

En atención a lo expuesto (Bélair et al., 2007) expresan que las competencias deben desarrollarse desde metodologías innovadoras y coherentes con procesos significativos que abarquen currículos alternativos y cambios permanentes, razón por la cual es importante destacar que las competencias de los graduados 
reflejan un proceso de autonomía individual y grupal, para conseguir efectos o resultados favorables en cuanto a los servicios y productos que aportan significativamente a la organización. En este sentido, se observa como las instituciones educativas plantean en sus currículos específicamente en sus Proyectos Educativos Institucionales, prácticas orientadas al desarrollo de habilidades con sentido laboral, en el que se privilegia al ser, saber y hacer, tal como se refleja en la tabla 1.

Tabla 1: Competencias desarrolladas desde el currículo educativo del sujeto

\begin{tabular}{|l|l|}
\hline $\begin{array}{l}\text { Las Competencias de las } \\
\text { dimensiones del ser humano }\end{array}$ & $\begin{array}{l}\text { Competencia organizacional/desde la relación } \\
\text { productiva }\end{array}$ \\
\hline Ser & $\begin{array}{l}\text { Interés, sentido, expectativa, apropiación de su } \\
\text { rol- quereres. }\end{array}$ \\
\hline Saber & $\begin{array}{l}\text { Dominio de conocimientos, manejo de relaciones } \\
\text { desde lo Inter, Intra y Socio grupal. }\end{array}$ \\
\hline Hacer & Aplicabilidad de lo aprendido. \\
\hline
\end{tabular}

En el plano internacional, (Anderson et al., 2014) afirman que la innovación debe considerarse como un replanteamiento de todas las capacidades, permitiendo la mejora continua de los egresados vistos como productos que se presentarán en el mercado, compitiendo bajo la mirada de los nuevos desafíos que enfrenta la enseñanza, desarrollando así líneas de acción que compitan en mercados laborales y configuren las competencias del ser, saber y hacer.

Por su parte, el propiciar la innovación pedagógica en los planes y programas académicos dependerá de la relación entre los modelos educativos actuales y las tendencias internacionales. Es por ello que el contribuir con una educación superior que esté en la búsqueda de ampliar las capacidades de desarrollo en el mejoramiento empresarial, superación y perfeccionamiento de la vida social y cultural, permitirá preparar al nuevo graduado con capacidad para direccionarse a nuevos caminos entre en alianzas con mercados laborales nacionales e internacionales. Reafirma (Vega, 2016) al considerar que las competencias de los profesionales deben ser perfeccionadas acorde a los requerimientos exigidos en el plano laboral y al desarrollo del talento humano.

\section{OTROS ANTECEDENTES}

EL artículo se centra en el abordaje de las concepciones teóricas de estudio de impacto, currículo, resignificación de la práctica pedagógica investigativa y el egresado frente a la empleabilidad. Por lo tanto, estos conceptos requieren de una breve descripción.

Estudio de impacto

La importancia que tiene la educación frente al impacto laboral, demanda que el profesional deba mostrar resultados que garanticen el servicio prestado y los posibles resultados desde lo personal y social; demostrando lo aprendido y superando el crecimiento y avance en cuanto a los conocimientos con la Universidad que lo formó. Existen dos variables importantes válidas acorde a lo antes esbozado, una de ellas es la influencia interna, donde se tiene en cuenta lo cultural, lo político, lo educativo, y la incidencia en cuanto al manejo y apropiación de los estudiantes, que de manera creativa demuestran sus competencias; la otra variable corresponde a lo externo, que responde a los desafíos presentados durante la vida, es decir, cómo los enfrenta y cómo resolverá los dilemas que a diario le depara la vida. Por tanto, el estudio de impacto representa el mejoramiento continuo del ser humano, en lo laboral, profesional y social, atendiendo a las dimensiones del ser, desde el punto de vista afectivo, cognitivo y expresivo.

\section{Currículo}

Bikfalvi et al. (2013) los currículos pueden utilizar metodologías tecnológicas, que permitan facilitar los auto reportes de los datos que estudian las competencias y el alcance que tienen sobre las necesidades de los estudiantes, indicando la flexibilidad académica con el desarrollo eficiente de los planes de estudios; contextualizados y ajustados con base a las competencias educativas. En este sentido, las Universidades tienen como objetivo evaluar a los egresados como su principal fuente de relación entre los principios y valores de la misma, siendo considerados como un profesional que dejará una huella inquebrantable al poner en alto a la Institución de Educación Superior donde egresó. Para (Icarte y Labate, 2016) los currículos se fortalecen cuando los planes de estudios se pactan de acuerdo a las necesidades de los estudiantes, desarrollando habilidades que permitan generar un saber en relación a la aplicación de experiencias que crean innovación en los contenidos programáticos. 
Bolliger y Martindale (2004) manifiestan que el agrado de los egresados frente al programa que los formó se traduce en la satisfacción entre la relación maestro y estudiante, características comunes de los grupos a los que perteneció, currículos académicos, formación del estudiante, servicios recibidos y dotación de la localidad infraestructural, razón por la cual, las Instituciones deben contemplar cada uno de éstos aspectos en sus fines misiones, considerando el papel fundamental que tienen para el bienestar de los estudiantes durante su proceso formativo.

En lo que respecta a los resultados de este tipo de estudios permitirán reevaluar los programas académicos, desarrollando la mejora continua que admita superar dificultades y contribuir para la toma de decisiones. También permitirá el fortalecimiento de la planeación curricular en todas las áreas académicas, la actualización de los planes de estudio e impacto en los mercados laborales. Por tanto, el estudio de seguimiento se considera un resorte de elevada importancia para todas las Universidades, pues el desempeño de los graduados constata variables desde el punto de vista profesional, personal y repunta a la calidad de la educación superior y la eficiencia de la misma.

Desde la mirada de (Cajiao, 2016) los estudios de seguimiento de los graduados de las diferentes carreras buscan en primera instancia, fortalecer los currículos educativos universitarios y en segunda instancia contribuir con el desempeño profesional en los campos laborales donde estos se desenvuelvan, atendiendo a la evolución de su perfil profesional y los campos de mercadeo laboral. Para (Blackwell, et al., 2005) los programas ofertados por las Universidades, donde los egresados se encuentran en grado de satisfacción, hace que ellos se sientan motivados a volver a la Institución y también la recomienden a otros profesionales por el servicio de calidad recibido. En consecuencia con lo expuesto, los estudios de seguimiento de los graduados deben atender lo social y lo académico, para que de esta manera puedan desempeñarse dentro de sus sitios de trabajo, reconociéndoles sus calidades académicas, profesionales y sus competencias acordes con la gestión que realizan.

El impacto laboral de los Especialistas en Estudios Pedagógicos hace referencia a la articulación de los programas de esta especialidad, en coherencia con lo exigido por el Sistema Educativo Colombiano que obedece a programas de calidad educativa para ofrecer mejores profesionales. De esta manera se pretende que los profesionales entren al progreso de competir entre los mejores. Por lo tanto, el Sistema Educativo se alista en apuntarle a uno de sus grandes indicadores como lo son las competencias, donde los profesionales logren un mejor servicio como es servir al país y a su entorno. Desde esta óptica para (Jackson, 2012) los lineamientos de la educación superior deben estar relacionados con los enfoques por competencias, los cuales en estas directrices de alta calidad mencionadas anteriormente, se inserten en los programas curriculares para establecer la correspondencia entre los elementos del currículo y el ejercicio profesional que cada uno ejerce.

Frente a lo anterior, es importante destacar la mirada que plantean (López y Martínez, 2014) al considerar que los docentes deben generar espacios de reflexión curricular, aclarando que son ellos los que desarrollan los procesos de enseñanza y propician los aprendizajes necesarios para potenciar la calidad en los procesos académicos; para que las metas, los campos de acción y los currículos académicos de la educación superior permitan la autonomía de las Instituciones, permitiendo fortalecer la calidad en el desarrollo de los aprendizajes. De igual manera, los sistemas educativos, están llamados a reflexionar sus enseñanzas, en torno a nuevas miradas, donde el pensamiento aclare las dinámicas integradoras, lo que implica que las instituciones educativas promuevan la crisis para tomar posturas frente a la educación y generar innovaciones que originen nuevos encuentros entre los estudiantes y la formación, para así desafiar pensamientos complejos. Deduciendo que son precisamente las universidades las que tienen la imperiosa labor de generar espacios constructivos con los actores del hecho educativo, en donde se gestione el conocimiento a fin de contribuir a un pensamiento complejo que oriente los currículos educativos.

En este orden (Perrenoud, 2008) afirma que para el desarrollo de las competencias se requiere que a los saberes se le otorgue una relación que los vincule con las prácticas sociales y la formación de competencias indispensables para la vida, entendiendo que se le den nuevas oportunidades al mayor número de ciudadanos. Frente a lo expuesto se requiere orientar programas de seguimiento a egresados, para que las políticas de trabajo y valoración quede tipificada en las condiciones de calidad en los currículos colombianos y se destaquen criterios correspondientes a los programas de egreso, atendiendo a las condiciones mínimas de calidad y las exigencias de rigor legal de las leyes planteadas.

\section{Resignificación de la Práctica pedagógica investigativa}

La resignificación invita al maestro a darle un sentido más significativo a su práctica pedagógica, frente al accionar diario del trabajo de aula. Guevara (2013) afirma en este sentido que la resignificación es la mediación para darle un alto sentido a la experiencia pedagógica que se le ofrece a los estudiantes. Es decir, 
corresponde a todas las acciones que realiza el maestro como principio motivacional, reflejado en una nueva forma de pensamiento y apropiación del proceso aprendizaje en el cual participan los estudiantes. La escuela como sitio micro político, está llamada a promover una educación, donde las competencias ciudadanas y democráticas sean la base para fortalecer estructuralmente la comprensión del ser humano para el logro de la ciudadanía y la democracia como bases pilares para una nueva comprensión del desarrollo integral.

Desde la mirada, la práctica pedagógica da cabida a la experiencia donde la reflexión posibilita la cultura del sistema; destacando al ser humano como ese ser individual, donde actúa en relación con el otro, en un marco educativo. Por lo tanto, la práctica pedagógica compromete al maestro dentro de su rol como un ser creador capaz de aportar experiencias innovadoras que estén encaminadas a facilitar mejores ambientes educativos con el propósito que este docente tenga como marco de referencia la enseñanza, la investigación y la pedagogía.

En este sentido (McMahon, 2013) reconoce que los currículos deben atender una fundamentación de ejes transversales configurados como parte fundamental en la educación, como lo son los contenidos culturales, necesarios para la vida y la convivencia del ser humano, desarrollado desde una práctica pedagógica que crea conocimientos y genera transformaciones en el currículo. Por lo tanto, éstos deben ser adaptados y ajustados a los alcances de las necesidades de los estudiantes evidenciados en los proyectos Institucionales. En cuanto a la práctica docente es concebida como aquella que muestra la verdadera relación entre maestro y estudiante frente al proceso de enseñanza y aprendizaje, evidenciando la pertinencia que tienen los educadores sobre el trabajo de aula.

Demostrando la apropiación que debe tener el docente y su disposición de actualizarse y perfeccionarse en sus métodos de enseñanza, para convertirse en ese facilitador del aprendizaje en un contexto determinado, deberá comprender, analizar, pensar y entender los diferentes objetivos y fundamentos que determinan la enseñanza. Para ello (Avendaño y Guacaneme, 2016) destacan la importancia de la escuela frente a la vanguardia de la globalización, que implica cambios mediante la adopción de nuevos retos para direccionarla en función de los objetivos propuestos, ampliando el rol pedagógico acorde a las exigencias y paradigmas de las reformas curriculares actuales.

\section{Egresado frente a la empleabilidad}

Como señalan (Fong et al., 2016), nos encontramos en una sociedad globalizante, en la que se experimentan cambios constantes a nivel organizacional. Se busca que la prioridad de la formación sea una constante donde los jóvenes logren puntos de encuentro desde la investigación, para poder afinar currículos que convergen en exigencias para entrar a competir con los requerimientos que plantea la educación; para que los planes de estudios y los mismos campos de la educación estén con las necesidades del mercado laboral. Lo que quiere decir que los cambios conllevan a la misma transformación del sistema educativo. Vale la pena destacar a (Villalta, 2015) cuando expresa que la implementación de las herramientas tecnológicas propicia el trabajo interactivo, que converge con el trabajo colaborativo, hasta el uso de nuevas tecnologías que van más allá de apegarse a la vanguardia de la práctica del nuevo recurso, que fortalecerán sin duda los aprendizajes y desenvolvimiento en su vida laboral.

En este sentido para (Didriksson, 2012) las Instituciones de Educación Superior, debe darse a la tarea de orientar sus programas académicos con procesos curriculares flexibles, donde sus acciones estén encaminadas a orientar y producir conocimientos en esta sociedad donde se rodea de situaciones complejas, y pueda competir en un mercado global, donde la empleabilidad deba convertirse en acciones dinámicas, para que las habilidades y destrezas de éstos sujetos, puedan mostrar sus saberes. Se hace necesario que puedan competir en otros mercados internacionales, para que así puedan exponer las fortalezas del programa educativo que los instruyó como profesionales y colocarlos a la altura de contextos externos, donde exigen individuos altamente competentes. La empleabilidad por su parte, requiere de unos factores de competencia, que sólo se logra, cuando se han desarrollado programas curriculares de alta calidad. Lo que se quiere es que la gestión para la empleabilidad, demande unas capacidades, factores y elementos, que exigen que se brinden oportunidades donde se conjuguen en las actitudes, intereses, motivaciones, conocimientos, formación y aptitudes para el posicionamiento de un mercado laboral de trabajo, abierto y flexible, donde se puedan subscribir.

\section{MÉTODOLOGÍA}

La presente investigación se enmarcó en el enfoque cuantitativo, ya que buscó demostrar los resultados obtenidos luego de la descripción de las variables y categorías relacionadas con el objeto de estudio. El tipo de estudio con el que se trabajó fue descriptivo explicativo: Los estudios descriptivos buscan caracterizar y especificar las propiedades importantes de personas, grupos, comunidades o cualquier otro fenómeno que 
sea sometido a análisis; registran, miden o evalúan diversos aspectos, dimensiones o componentes de los fenómenos a investigar (Briones, 2012). La muestra se conformó con los egresados de la Especialización en Estudios Pedagógicos durante los años 2014 al 2017. Con ellos se realizó un muestreo aleatorio simple estratificado. Se tomó una muestra del 99\%, equivalente a 247 especialistas egresados de los años 2014 hasta el 2017, los cuales participaron de manera voluntaria. Para los empleadores (Instituciones Educativas) donde laboran los egresados fue una muestra por conveniencia.

Se utilizaron técnicas cualitativas y cuantitativas, dirigidas a Graduados y Empleadores. Se trabajó con el cuestionario de auto reporte a profundidad, para cada población objeto de estudio, el cuestionario contenía preguntas múltiples distribuidas en 6 partes con un total de 21 ítems, el cual fue presentado ante jueces expertos para su validación. Para el análisis y procesamiento de la información se siguieron los siguientes pasos: Actualización del directorio de egresados, elaboración del directorio de Empleadores (Instituciones Educativas), elaboración, validación y pilotaje (ajuste) de los instrumentos dirigido a las poblaciones de: graduados y empleadores, aplicación de los instrumentos y procesamiento de la información. Finalmente se presentaron los resultados mediante la utilización de gráficos circulares y de barras con sus respectivas proporciones porcentuales.

\section{RESULTADOS}

Para el análisis de la información y procesamiento de la información cuantitativa se utilizó el programa estadístico Statistical Package for Social Sciencies (SPSS v. 11.0 para Windows). Y para lo cualitativo se abordó a través del análisis discursivo, el cual se refiere a la interpretación discursiva de los datos sin aplicar técnica. A continuación, se exponen los resultados encontrados en el estudio de impacto de los graduados de la especialización de estudios pedagógicos, frente a la investigación, resignificación, práctica pedagógica, su percepción y la de los empleadores. Se tomó una muestra del 99\%, equivalente a 247 especialistas egresados de los años 2014 hasta el 2017. Ver figura 1.

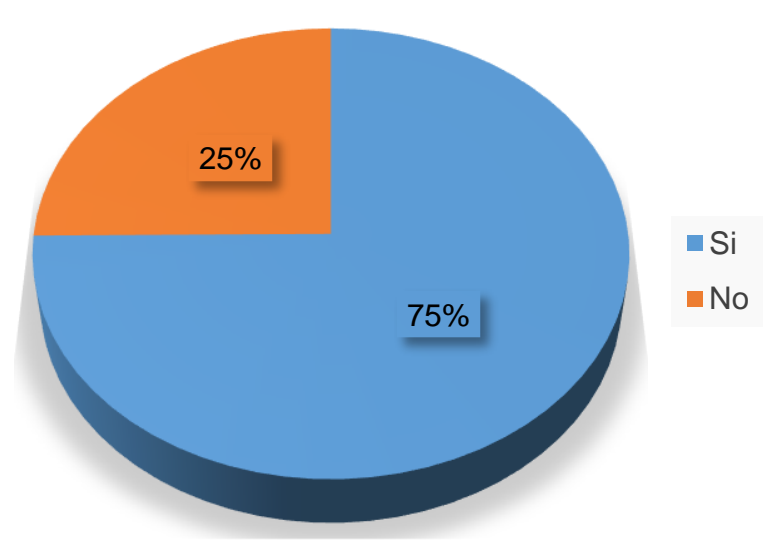

Fig. 1: Empleadores

La figura 1 denominada Empleadores, se relaciona el nivel de consideración del egresado de la Especialización de Estudios Pedagógicos de la Universidad de la Costa con el mejoramiento de sus prácticas pedagógicas al finalizar su formación académica, donde el 75\% de los empleadores expresaron que si existe un nivel de consideración en cuanto al mejoramiento de sus prácticas, al notarse innovación y transformación en el trabajo laboral de su día a día, cambiando el trato con sus estudiantes y el estilo de orientación de las actividades pedagógicas. Esto fue evidente una vez finalizó el egresado su formación académica. En lo que respecta al $25 \%$ de los empleadores, manifestaron que no existe un nivel de consideración frente al proceso de sus prácticas, debido a que el egresado una vez culminado su formación académica, el trato con sus estudiantes se mantuvo igual, es decir no tuvo ninguna mejoría.

Por otra parte (Buckingha y Ferguson, 2012) señalan que una sociedad globalizante, exige de profesionales altamente comprometidos que estén al alcance de los sistemas multimedia. Este análisis, permitió hacer una comparación frente a lo argumentado por (Kotsifos et al., 2013) que consideran la habilidad académica, el fortalecimiento de los programas, el nombre de la Institución, las habilidades aprendidas en la formación recibida y la visión de los empleadores con respecto a las capacidades desarrollados por los egresados, como indicadores relevantes para ser escogidos por organizaciones con altas exigencias en el mercado laboral. Ver figura 2. 


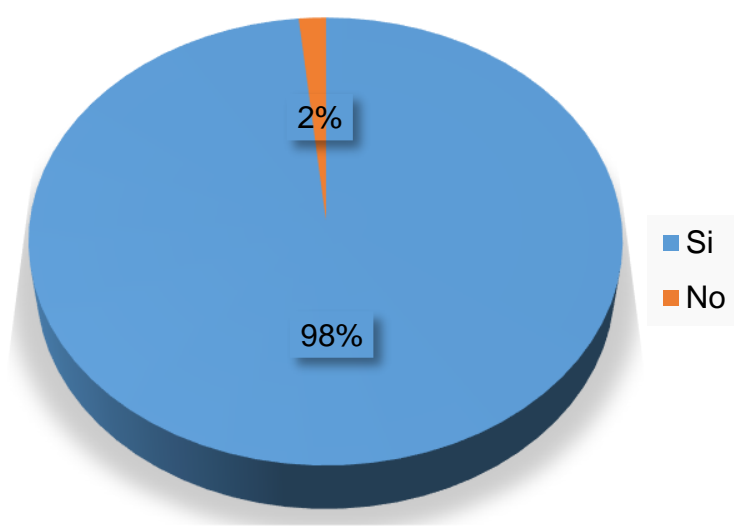

Fig. 2: Currículos y condiciones laborales

En relación a la figura 2 de nombre Currículos y condiciones laborales, se observa que el $98 \%$ de los egresados se encuentran laborando y sólo el $2 \%$ aun no cuentan con empleo, éste porcentaje equivalente a 4 especialistas, lo que indica que la Universidad se ha preocupado por llevar un currículo que atienda a las competencias laborares de estos egresados. Y para la (International Labour Organization, 2000) las competencias determinan en los profesionales las formas de acceso a la vida laboral, lo que indica que cada vacante que se presente en una empresa será reemplazada por un profesional que tenga competencias definidas. Ver figura 3.

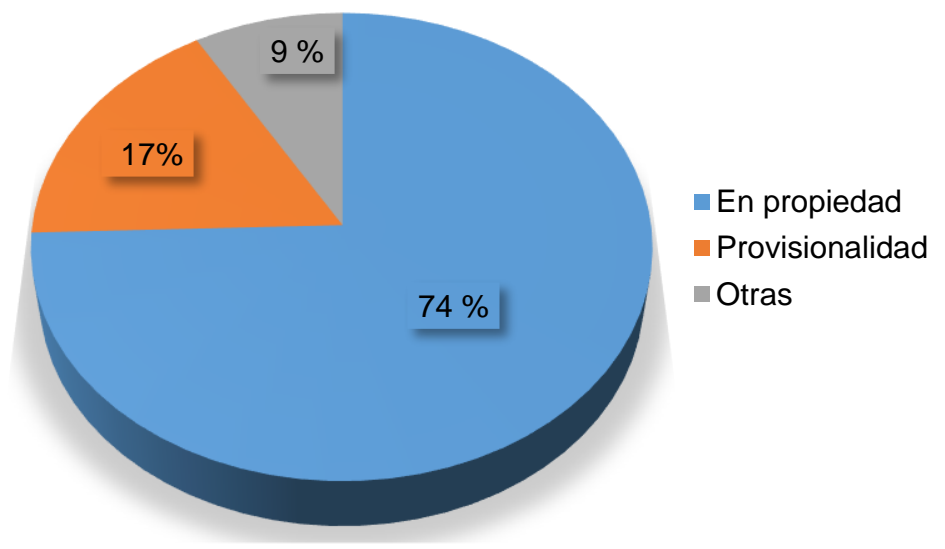

Fig. 3: Tipo de contratación y/o vinculación-(empleabilidad)

Siguiendo con la figura 3 relacionada con la variable contratación y/o vinculación, el 74\% que equivale a 184 egresados están vinculados en propiedad, 42 egresados de la misma profesión correspondiente al $17 \%$ se encuentra vinculado en provisionalidad y sólo 21 Especialistas egresados correspondiente al $9 \%$, se encuentran en otras actividades diferentes a las educativas. Lo que quiere decir que un porcentaje alto se encuentra vinculado al servicio educativo en propiedad. Ver figura 4.

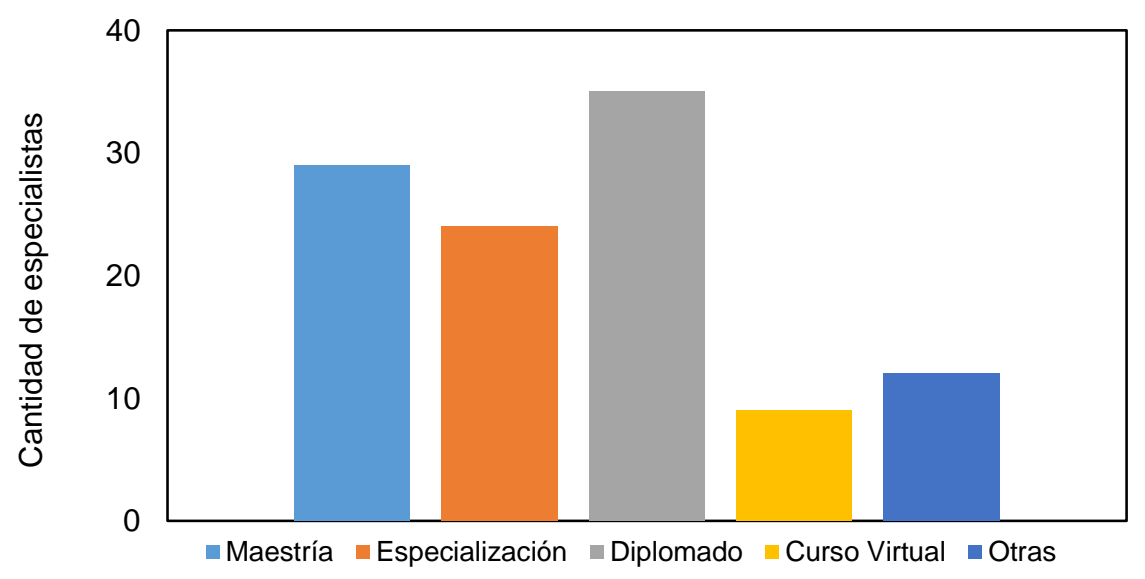

Fig. 4: Otros estudios realizados 
En cuanto a lo correspondiente a la figura 4 referida a Otros estudios realizados, se observa que el $12 \%$ correspondiente a 29 Especialistas han realizado estudios de Maestría, por su parte, el 64\% representados en 35 graduados han realizado diplomados, el 15\% correspondientes a 9 Especialistas han estudiado cursos virtuales y el $4 \%$ de 12 Especialistas otros tipos de estudios diferentes a los mencionados. Tal como lo expresa (Imafidon, 2015) los estudios de un profesional se vuelven resignificativos cuando sus conocimientos adquiridos los colocan al servicio de su comunidad, mostrando sus competencias profesionales en los contextos empresariales. Ver figura. 5

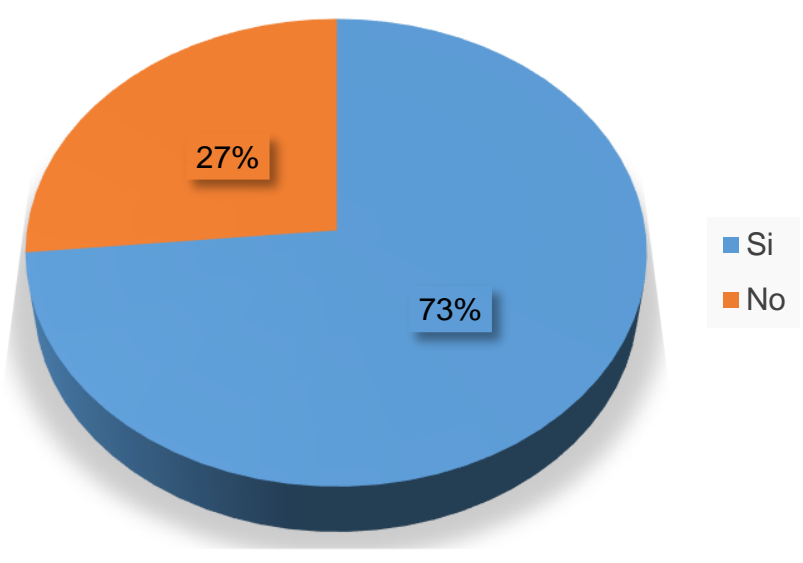

Fig. 5: Prácticas pedagógicas e investigativas

En lo referente a la figura 5 denominada Prácticas pedagógicas e investigativas, en orientación de procesos de formación en ambientes presenciales y virtuales de aprendizaje, 183 egresados expresaron que sí realizan prácticas pedagógicas e investigativas, tendientes a la orientación de procesos en ambientes presenciales y virtuales de aprendizaje, con un porcentaje del 73\% y sólo 66 egresados expresaron que no orientan prácticas pedagógicas e investigativas en ambientes virtuales de aprendizaje, con un porcentaje del $27 \%$. La práctica pedagógica genera transformación en la manera como se media el acto educativo. En cuanto a la percepción que tienen los graduados con su programa académico, se logró evidenciar cuál es el nivel considerado. Ver figura 6 .

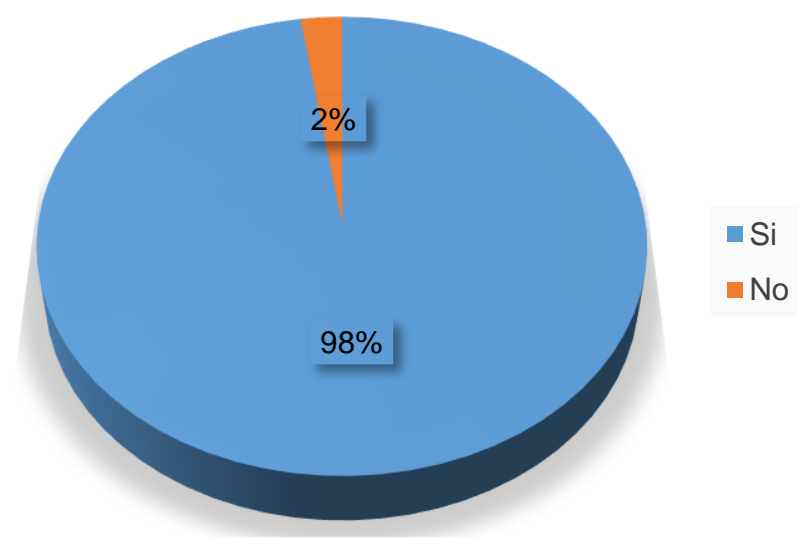

Fig. 6: Calidad académica del programa

Siguiendo con la figura 6 referido a la calidad académica del programa, en cuanto al nivel de fortalecimiento en su desempeño de buenas prácticas académicas, 243 egresados expresaron que sí hay niveles de fortalecimiento en sus prácticas pedagógicas, con un 98\%, para los programas académicos y 4 egresados expresaron que no tienen correspondencia, con un equivalente del $2 \%$. Para (CNA ,2014) es importante que la Instituciones de Educación Superior, inserten los lineamientos claves de alta calidad, y los articulen con las exigencias de los programas curriculares, atendiendo lo que piden las competencias laborales para los mercados internacionales.

\section{CONCLUSIONES}

De acuerdo al trabajo presentado y a los resultados obtenidos, se pueden plantear las siguientes conclusiones principales: 1) desde los propósitos curriculares de la Universidad de la Costa, específicamente en el programa de Especialización en Estudios Pedagógicos, los egresados si mejoraron la calidad de las prácticas 
pedagógicas en sus Instituciones; 2) una gran minoría de los egresados tienen resistencia al cambio para la mejora de sus prácticas pedagógicas, pues el trabajo de aula sigue un rumbo igual, sin manejo investigativo y sin apoyo en ambientes virtuales de aprendizajes; 3) se observó la existencia de un alto nivel de percepción de los empleadores con respecto a la calidad de formación recibida del egresado y 4) las condiciones de calidad del programa ofertado en la especialización es un factor determinante en la cualificación del egresado frente al ejercicio de su práctica, generando así una considerable mejora en su desempeño laboral. De este modo, constituye un punto de partida muy sólido para los procesos internos de mejoramiento institucional y se convierte en un referente claro para que los usuarios potenciales de la Educación Superior puedan hacer una elección suficientemente informada.

\section{REFERENCIAS}

Abadía, A.R., Bueno, C. y otros cinco autores, Competencias del Buen Docente Universitario: Opinión de los estudiantes, Revista de docencia Universitaria, 13(2), 363-390 (2015).

Anderson N., Potocnik, K. y Zhou, J., Innovation and Creativity in Organizations:Astate-Of-The-Science Review, Prospective Commentary, and Guiding Framework, Journal of Management, 40 (5),1297-1333 (2014).

Avendaño, W.R. y Guacaneme, R.E., Educación y Globalización una Visión Crítica, Civilizar Ciencias Humanas y Sociales, 16 (30), 191-206 (2016).

Bélair, L., Laveault, D. y Lebel, C., Les Compétences Professionnelles en Enseignement et Leur Évaluation, 1-272, Presses de l'Université d'Otawa, Otawa, Canadá (2007).

Beneitone, P., Esquetini, C. y otros cuatro autores, Reflexiones y Perspectivas de la Educación Superior en América Latina: Informe Final Proyecto Tuning América Latina: 2004-2007,1- 429, Universidad de Deusto, Bilbao, España (2007).

Bikfalvi, A.; Rafart, A. y Manceb, N., The Assessment of Competence in University Adult Education: A Dynamic Approach, International Journal of Management Knowledge and Learning, 1(2), 3-22 (2013).

Blackwell, R., Miniard, P. y Engel, J., Consumer Behaviour: an Asia Pacific Approach,10ª Ed., 1-832, South-Western College Pub, California, EEUU (2005).

Bolliger, D.U. y Martindale, T., Key Factors for Determining Student Satisfaction in Online Courses, International Journal on E-learning, 3(1), 61-67 (2004).

Bravo,N., Competencias Proyecto Tuning-Europa, Tuning.-America Latina (2007).

Briones, G., Metodología de la Investigación Cuantitativa en las Ciencias Sociales, Instituto Colombiano para el Fomento de la Educación Superior-ICFES, 18-22, Bogotá, Colombia (2012).

Buckingham, S. y Ferguson, R., Social Learning Analytics, Educational Technology \& Society, 15 (3), 3-26 (2012).

Cajiao, F., La Investigación en los Procesos de Formación, Gestión Ingenio y Sociedad, ISSN: 2500-7718, 1(1), 1-3 (2016).

CNA., Lineamientos para la Acreditación de Programas de Pregrado, 8-10, Bogotá, Colombia (2014).

Didriksson, A., The New Transformation Agenda in Latinamerica Higher Education, Perfiles Educativos, 34(138), 184-203 (2012).

Fong, W., Acevedo, R.L., y Severiche, C.A., Estrategia de Investigación Formativa Tecnológica: El Caso del Proyecto Integrador, Itineraria Educativo, 67, 103-121 (2016).

Guevara, M., Una Práctica Reconstruida a partir de la Reflexión Docente, El Astrolabio, ISSN: 0124-213X, 12(2), 129-135 (2013).

Icarte, G. y Labate, H., Metodología para la Revisión y Actualización de un Diseño Curricular de una Carrera Universitaria Incorporando Conceptos de Aprendizaje Basado en Competencias, https://doi.org/10.4067/S0718-50062016000200002, Formación Universitaria, 9(2), 03-16 (2016).

International Labour Organization., World Labour Report 2000. Income Security and Social Protection in a Changing World, $1^{\text {a }}$ Ed, 14-16, OIT, Ginebra, Suiza (2000).

Imafidon, C., Social Responsibility, Quality of Work Life and Motivation to Contribute in the Nigerian Society, Journal of Business Ethics, 126, 219-233 (2015).

Jackson, D., Business Undergraduates' Perceptions of their Capabilities in Employability Skills: Implications for Industry and Higher Education, https://doi.org/10.5367/ihe.2012.0117, Industry and Higher Education, 26(5), 345-356 (2012).

Kotsifos, V, Alegakis, A. y Philalithis, A., Listening to and Learning from Graduate's Perceptions: Implications for Change?, International Education Studies, 6(7), 189-199 (2013).

McMahon, J.P., Applied Genre Analysis: The Case of Introductions in Spanish Teacher Trainees Curricular Design Documents, Revista Española de Lingüística Aplicada, (1), 165-182 (2013).

Orozco, J.C., Olaya, A. y Villate, V., ¿Calidad de la Educación o Educación de Calidad? Una Preocupación más allá del Mercado. Revista Iberoamericana de Educación, 51, 161- 181 (2009). 
Perrenoud, P., Construir las Competencias ¿Es Darle la Espalda a los Saberes?, Revista de Docencia Universitaria, 6(2), 1-16 (2008).

Rojo, R. y Navarro, N., Competencias Genéricas Adquiridas, según Estudiantes de una Carrera de la Salud, https://doi.org/10.1016/j.riem.2016.02.003, Investigación en educación médica, 5(19), 172-181 (2016).

López, M. y Martínez, N. D., Gestión Curricular Base de Calidad Académica, Revista Experiencia Docente, 1(1), 9-20 (2014).

Vega, M., Neocompetencias, Nuevo Enfoque de Competencias Laborales en Salud Ocupacional. Educ Med Super, 30 (3), 627-638 (2016).

Villalta, P., Pedagogical Processes and Use of Technology in the Classroom, Revista Complutense de Educación, 26(2), 405-4024 (2015). 